\title{
Fabry-Pérot Oscillations in Correlated Carbon Nanotubes
}

\author{
W. Yang $\odot,{ }^{1,2}$ C. Urgell, ${ }^{1}$ S. L. De Bonis $\odot,{ }^{1}$ M. Margańska $\odot,{ }^{3}$ M. Grifoni $\odot,{ }^{3}$ and A. Bachtold ${ }^{1}$ \\ ${ }^{1}$ ICFO-Institut De Ciencies Fotoniques, The Barcelona Institute of Science and Technology, \\ 08860 Castelldefels (Barcelona), Spain \\ ${ }^{2}$ Beijing National Laboratory for Condensed Matter Physics and Institute of Physics, Chinese Academy of Sciences, \\ Beijing 100190, People's Republic of China \\ ${ }^{3}$ Institut für Theoretische Physik, Universität Regensburg, D-93040 Regensburg, Germany
}

(Received 17 March 2020; accepted 24 September 2020; published 27 October 2020)

\begin{abstract}
We report the observation of an intriguing behavior in the transport properties of nanodevices operating in a regime between the Fabry-Pérot and the Kondo limits. Using ultrahigh quality nanotube devices, we study how the conductance oscillates when sweeping the gate voltage. Surprisingly, we observe a fourfold enhancement of the oscillation period upon decreasing temperature, signaling a crossover from singleelectron tunneling to Fabry-Pérot interference. These results suggest that the Fabry-Pérot interference occurs in a regime where electrons are correlated. The link between the measured correlated Fabry-Pérot oscillations and the SU(4) Kondo effect is discussed.
\end{abstract}

DOI: 10.1103/PhysRevLett.125.187701

Electron interactions and quantum interference are central in mesoscopic devices. The former are due to the electronic charge and give rise to many-body effects; the latter emerges due to the wavelike properties of an electron. Resonant ballistic devices with a few conduction modes and moderate coupling to electrodes are sensitive to both of these electronic properties. On one hand, quantum interference between electron waves backscattered at the boundaries between the mesoscopic system and the metallic electrodes gives rise to resonant features in the transmission, analogous to the light transmission in an optical Fabry-Pérot cavity [1]. On the other hand, if the electron spends enough time in the mesoscopic device before being transmitted, Coulomb repulsion can also become important, giving rise to Coulomb blockade and single-charge tunneling effects [2]. Despite considerable efforts, the interplay between electron interactions and quantum interference remains poorly understood from both an experimental and a theoretical point of view due to the many-body character of the problem. This is the topic of the present Letter.

Carbon nanotubes (CNTs), semiconducting nanowires, and edge channels of the quantum Hall effect are ideal quasi-one-dimensional (1D) systems to study both electron correlations and quantum interference. In fact, various many-body effects, including Coulomb blockade [3-5], Wigner phases [6-9], and Kondo physics [10-21], as well as Fabry-Pérot and Mach-Zehnder oscillations resulting from electron interference [22-28], have been observed in these multimode 1D systems. It is possible to switch from interaction- to interference-governed transport regimes by tuning the tunnel couplings at the interface between the wire and the electrodes, $\Gamma_{S}$ and $\Gamma_{D}$, for the source $(S)$ and drain $(D)$ electrodes. Which transport regime dominates crucially depends on how large the tunneling broadening $\hbar \Gamma=\hbar\left(\Gamma_{S}+\Gamma_{D}\right)$ is compared to other energy scales, in particular, to the charging energy $E_{C}$, being the electrostatic cost to add another (charged) electron to the wire [29]. In the so-called quantum dot limit, characterized by $\hbar \Gamma \ll E_{C}$, tunneling events in and out of the wire are rare and Coulomb charging effects are dominant. They give rise to Coulomb blockade phenomena and incoherent singleelectron tunneling in the regime $\hbar \Gamma<k_{B} T \ll E_{C}$. By decreasing temperature, one expects coherent single-electron tunneling for $k_{B} T \simeq \hbar \Gamma \ll E_{C}$, where the width of the Coulomb peaks is determined by $\Gamma$; at even lower temperatures, when spin-fluctuations become relevant, the Kondo effect emerges as the dominant transport mechanism. In the opposite limit of large transmission, $\hbar \Gamma \gg E_{C}$, interference effects give rise to the characteristic Fabry-Pérot patterns, which can be easily calculated from a noninteracting single-particle scattering approach [22]. The focus of this Letter is the intermediate transmission regime $\hbar \Gamma \sim E_{C} \gg$ $k_{B} T$ when no clear hierarchy of energy scales exists.

An experimental hallmark of both interaction- and interference-dominated transport is the modulation of the conductance when sweeping the electrochemical potential, that is, by varying the gate voltage $V_{g}$. In the incoherent tunneling regime, the alternance of single-electron tunneling and Coulomb blockade physics results in finite conductance peaks with a period in $V_{g}$ of about $e / C_{g}$ [2], where $-e$ is the (negative) electron charge, and $C_{g}$ is the capacitance between the nanotube and the gate electrode; see Fig. 1(a). In contrast, in the interference-dominated regime, the conductance modulation of the FabryPérot oscillations arises from the electron wave phase 
(a)
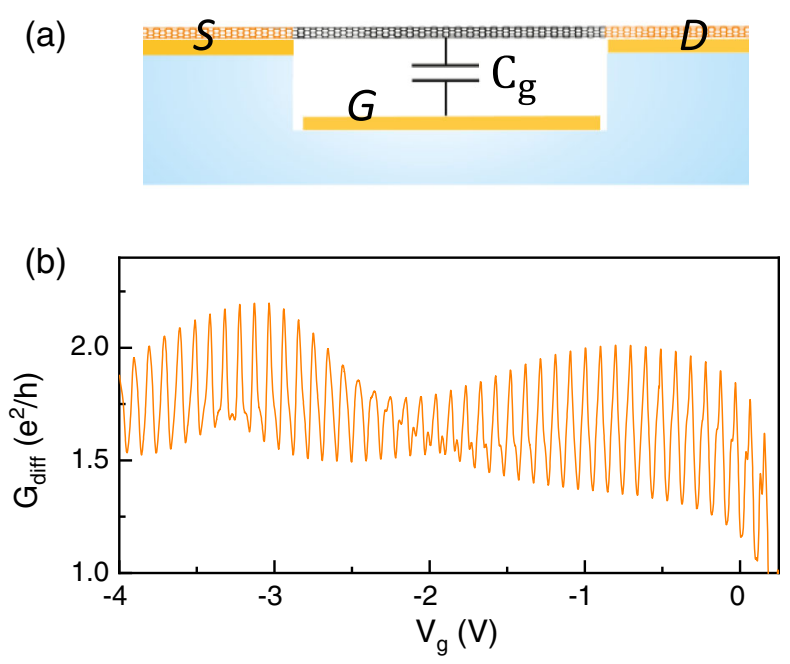

FIG. 1. Schematics of the device and low-temperature transport characteristics. (a) The three-terminal device with a suspended CNT contacted to source $(S)$, drain $(D)$, and gate $(G)$ electrodes. (b) Gate voltage dependence of the conductance at zero-sourcedrain voltage of device I at $T=15 \mathrm{mK}$. An oscillating voltage with amplitude smaller than $k_{B} T / e$ is applied to measure the differential conductance.

accumulated during a round trip along the wire. The presence of valley and spin degrees of freedom in CNTs gives rise to interferometers with oscillation period $\Delta V_{g}=$ $4 e / C_{g}[22]$.

In this Letter, we improve the quality of nanotube devices to an unprecedented level. We discover a crossover of the conductance oscillation period between $e / C_{g}$ and $4 e / C_{g}$ upon sweeping temperature. Above liquid helium temperature, the period is $e / C_{g}$ with oscillation amplitudes pointing to coherent single-electron tunneling in an open quantum dot configuration. At low temperature, the period becomes $4 e / C_{g}$, and the oscillations feature typical characteristics of Fabry-Pérot interference. These unexpected data are a clear signature of the interplay between interaction and quantum interference.

Experimental results. - We grow nanotubes by chemical vapor deposition on prepatterned electrodes [30]. The nanotube is suspended between two metal electrodes; see Fig. 1. We clean the nanotube in the dilution fridge at base temperature by applying a high constant sourcedrain voltage $V_{\text {sd }}$ for a few minutes (see Sec. I of the Supplemental Material [31]). This current-annealing step cleans the nanotube surface from contamination molecules adsorbed when the device is in contact with air. The energy gap of the two nanotubes discussed in this Letter is on the order of $10 \mathrm{meV}$ (for details, see the Supplemental Material [31]). The length of the two suspended nanotubes inferred by scanning electron microscopy (SEM) is about $1.5 \mu \mathrm{m}$.

Figure 1(b) shows the modulation of the differential conductance $G_{\text {diff }}$ of device I as a function of $V_{g}$ in the hole-side regime at $15 \mathrm{mK}$. Rapid conductance oscillations are superimposed on slow modulations. Since the conductance remains always large, that is, above $e^{2} / h$, we attribute the rapid oscillation to the Fabry-Pérot interference with period in gate voltage being $\Delta V_{g}=4 e / C_{g}$. The slow modulation may be caused by the Sagnac interference $[25,26]$, the additional backscattering due to a few residual adatoms on the CNT, the symmetry breaking of the electronic wave function by the planar contacts of the device, or any combination of these (for further discussion, see Sec. I and II A of the Supplemental Material [31]).

A crossover to a regime dominated by the charging effect in an open interacting quantum dot is observed upon increasing temperature. Specifically, by sweeping the temperature from $15 \mathrm{mK}$ to $8 \mathrm{~K}$, the amplitude of the oscillations gets smaller. Further, the oscillation period gets four times lower, changing from $4 e / C_{g}$ at $15 \mathrm{mK}$ to $e / C_{g}$ at $8 \mathrm{~K}$; see Figs. 2(a) and 2(c)-2(e). The period in $V_{g}$ is calibrated in units of $e / C_{g}$ using the measurements in the electron-side regime, where regular Coulomb oscillations are observed at $8 \mathrm{~K}$, as shown in Fig. 2(b). The same behavior is observed in device II; Figs. 3(a) and 3(b). The $4 e / C_{g}$ oscillations vanish at $\sim 3 \mathrm{~K}$ in both devices, whereas the $e / C_{g}$ oscillation amplitude is suppressed to almost zero below $\sim 1 \mathrm{~K}$ in device I and below $\sim 0.1 \mathrm{~K}$ in device II; see Figs. 2(f) and 3(b).

Our interpretation of a temperature-induced crossover between two seemingly distinct transport regimes is confirmed by measured maps of the differential conductance as a function of source-drain and gate voltages at $T=15 \mathrm{mK}$ and $T=8 \mathrm{~K}$, as shown in Figs. 4(a) and 4(d), respectively. The low-temperature data feature the regular chess-boardlike Fabry-Pérot interference pattern [22], while the high-temperature data show smeared Coulomb diamonds. Such measurements further allow us to extract important energy scales for our device. The characteristic bias $V_{\text {sd }}^{*}$ indicated by the arrow in Fig. 4(a) yields a singleparticle excitation energy $\Delta E=e V_{\mathrm{sd}}^{*} \simeq 1.7 \mathrm{meV}$. This value is consistent with what is expected from a nanotube with length $L \simeq 1.5 \mu \mathrm{m}$. Assuming the linear dispersion $\varepsilon(k)=\hbar v_{F} k$, with longitudinal quantization $k_{n}=n \pi / L$ and the Fermi velocity $v_{F}=10^{6} \mathrm{~m} / \mathrm{s}$, it yields $\Delta E=\varepsilon\left(k_{n+1}\right)-\varepsilon\left(k_{n}\right)=\hbar v_{F} \pi / L \simeq 1.4 \mathrm{meV}$. The charging energy is estimated from the charge stability diagram measurements at $8 \mathrm{~K}$, Fig. 4(d); from the Coulomb diamond, indicated by the dashed lines, a charging energy $E_{C} \simeq 3.6 \mathrm{meV}$ is extracted. Further, we estimate $\hbar \Gamma \sim E_{C}$ because of the strong smearing of the diamonds in Fig. 4(d) and the weak conductance modulation at $8 \mathrm{~K}$ in Fig. 2(a). The energy hierarchy in our experiment is thus $E_{C} \simeq \hbar \Gamma \simeq \Delta E \gg k_{B} T$.

The evolution of the $15 \mathrm{mK}$ conductance oscillations as a function of the source-drain bias shows that both oscillations coexist over a large bias range, albeit with modulated strengths; see Figs. 4(a)-4(c). The main trend is that 

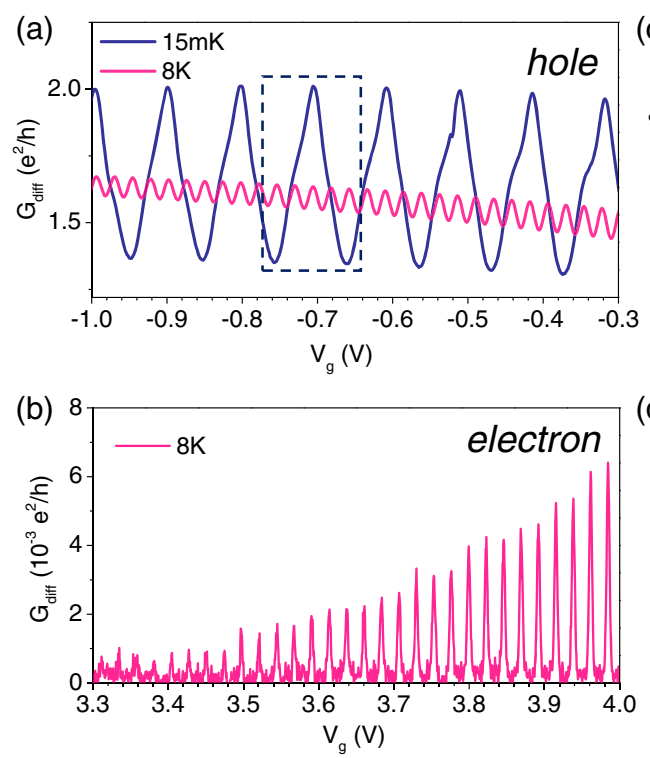
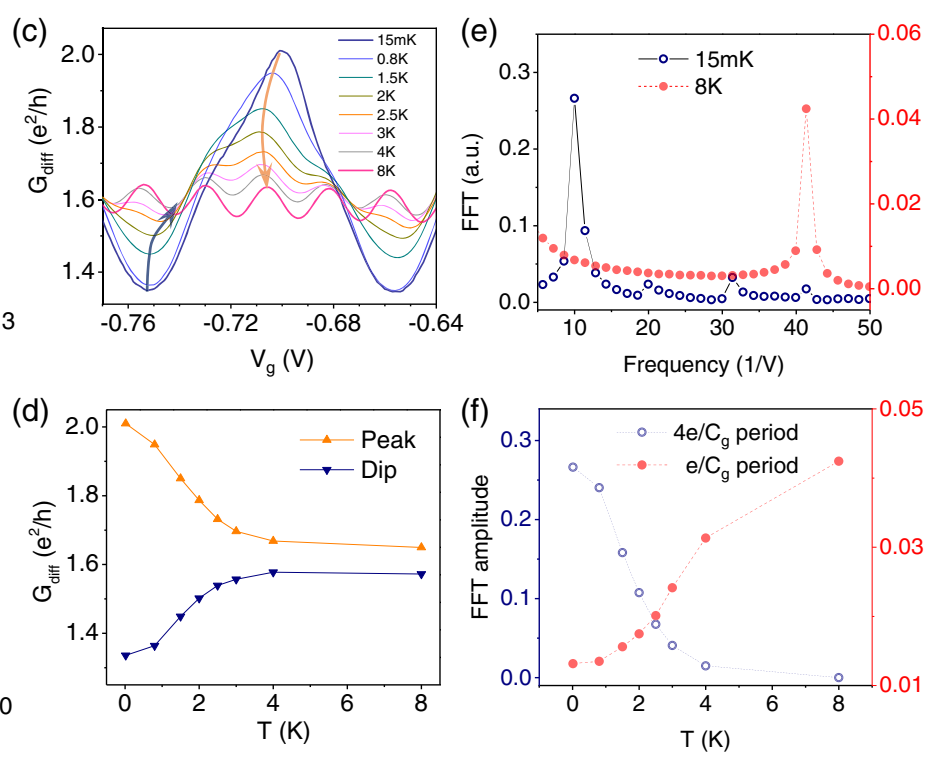

FIG. 2. Temperature-induced crossover from an interference-dominated to a charging-controlled regime in device I. (a), (b) Oscillations of the conductance $G_{\text {diff }}$ versus gate voltage $V_{g}$ in the hole- and electron-doped regimes. (c) Evolution of the oscillation period for a series of different temperatures. The range of $V_{g}$ shown in this figure is highlighted in panel (a) by a dashed rectangle. (d) Temperature dependence of the conductance associated with a peak and a dip, as indicated by arrows in (c). (e) Fast Fourier transform (FFT) of the $G_{\text {diff }}\left(V_{g}\right)$ traces at $15 \mathrm{mK}$ and $8 \mathrm{~K}$ measured for $V_{g}$ between $-1.0 \mathrm{~V}$ and -0.3 V. (f) Temperature dependence of the FFT amplitude associated with the $4 e / C_{g}$ period oscillations and the $e / C_{g}$ period oscillations.

the oscillation period changes from $4 e / C_{g}$ at zero bias to $e / C_{g}$ at high bias. By contrast, the evolution in the perpendicular magnetic field shows that the conductance peaks are split in two, with the splitting in gate voltage being linear in magnetic field; see Figs. 3(c) and 3(d).
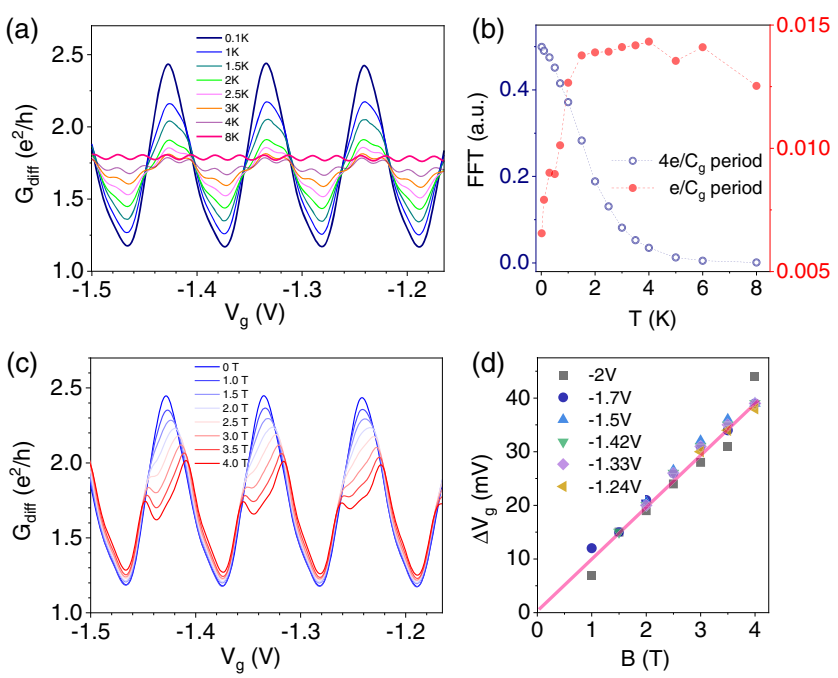

FIG. 3. Measurements on device II. (a) Conductance traces for a series of different temperatures. (b) Temperature dependence of the FFT amplitude associated with the $4 e / C_{g}$ and the $e / C_{g}$ period oscillations. (c) Conductance traces for different perpendicular magnetic fields at $15 \mathrm{mK}$. (d) Peak splitting as a function of magnetic field for the conductance peaks at different gate voltages.
This is attributed to the Zeeman splitting, since the associated $g$ factor is $2.4 \pm 0.4$. The error in the estimation arises from the uncertainty in the lever arm. These data indicate degeneracy of the four electron levels associated to the spin and valley degrees of freedom.

Discussion.-We examine possible origins of the temperature-induced period change. Let us first assume that interactions are not important. Then, upon lowering temperature, noninteracting Fabry-Pérot oscillations are expected to emerge when the thermal smearing becomes smaller than the single-particle excitation energy. However, thermal smearing is associated to a characteristic temperature $T_{\text {th }} \sim \Delta E / k_{B} \approx 20 \mathrm{~K}$, which is rather different from the measured crossover temperature $T_{C} \sim 3 \mathrm{~K}$ in Figs. 2(f) and $3(\mathrm{c})$. In addition, thermal smearing cannot explain the emergence at temperatures above $T_{C}$ of the $e / C_{g}$ oscillations due to coherent single-electron tunneling. Therefore, thermal decoherence is not at the origin of the measured period change. This is further supported by single-particle Fabry-Pérot interference calculations, based on an accurate tight-binding modeling of CNTs, that we carried out. We also considered the complementary regime and investigated whether charge fluctuations could be the cause of our finding. However, when using an interacting multilevel quantum dot with fourfold degenerate energy levels in the regime $E_{C} \simeq \hbar \Gamma$, we could not reproduce the measured fourfold variation of the period. Both the single-particle and the interacting calculations are described in the Sec. II of the Supplemental Material [31]. 


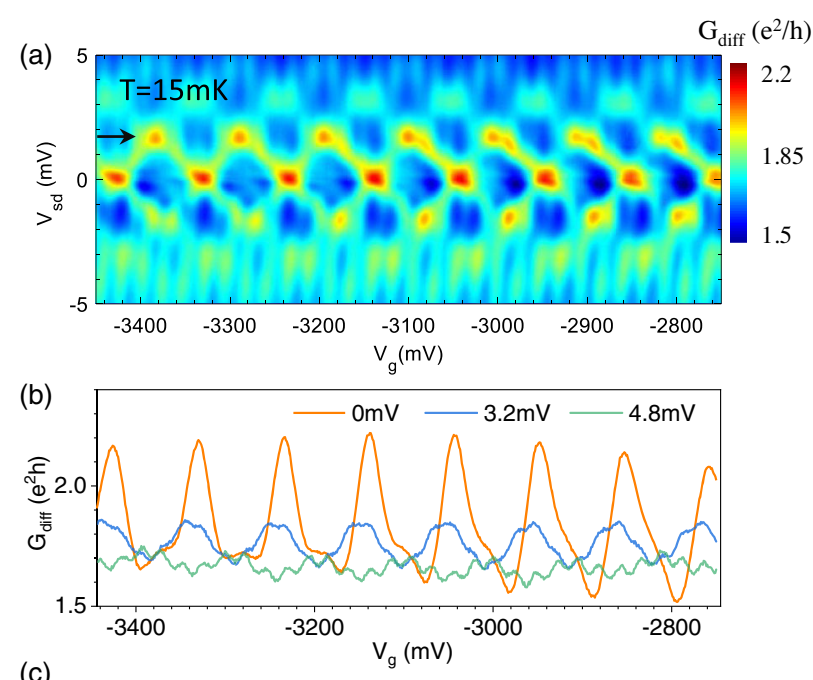

and valley fluctuations [11]. This results in a crossover from charging effects at high temperature to the increased conductance of Kondo resonances at zero temperature, with a fourfold enhancement of the oscillation period $[13,29,38]$. In contrast to our observations, though, in the SU(4) Kondo effect, the conductance alternates between large values close to $4 e^{2} / h$ at oscillation maxima and almost zero at minima [18,38]; see also Sec. Ib of the Supplemental Material [31]. In our annealed devices, the tunneling coupling is large; $\hbar \Gamma \simeq E_{C}$. The charge is no longer strongly localized within the dot. As a result, our devices are in a regime where there are also charge fluctuations in the nanotube, in addition to spin and valley fluctuations. This might be at the origin of the crossover of the conductance oscillation period observed in this Letter, similar to what happens in the SU(4) Kondo regime $[13,29,38]$, but with conductance minima clearly distinct from zero. We emphasize that the zero-source-drain bias, low-temperature $G_{\text {diff }}\left(V_{g}\right)$ data alone do not allow one to distinguish between noninteracting and correlated FabryPérot oscillations. However, the smooth modulation between $e / C_{g}$ and $4 e / C_{g}$ oscillations upon increasing the bias [see Fig. 4(c)] further supports our hypothesis of correlated Fabry-Pérot regime.

Conclusion.-Our Letter provides a comprehensible phenomenology of transport in nanotubes when both interference and interaction are involved. The findings presented in this Letter have been possible thanks to the high quality of the devices, since otherwise disorder leads to irregular $G_{\text {diff }}\left(V_{g}\right)$ modulations that are difficult to interpret. The main results are summarized as follows:

FIG. 4. From Fabry-Pérot patterns to blurred Coulomb diamonds in device I. (a) Map of the differential conductance as a function of $V_{\mathrm{sd}}$ and $V_{g}$ at $15 \mathrm{mK}$. From the position of the arrow, the single-particle excitation energy is extracted. (b) Differential conductance traces for a series of different source-drain voltages at $15 \mathrm{mK}$. (c) Source-drain voltage dependence of the FFT amplitude associated with the $4 e / C_{g}$ and the $e / C_{g}$ period oscillations at $15 \mathrm{mK}$. The curves are obtained by doing a FFT of the $G_{\text {diff }}\left(V_{q}\right)$ trace for each $V_{\text {sd }}$ value. (d) A map of the differential conductance as a function of $V_{\mathrm{sd}}$ and $V_{g}$ at $8 \mathrm{~K}$. The dashed lines highlight the contours of the Coulomb diamonds.

The high-temperature measurement of the charging effect in an open quantum dot indicates electron correlation. When reducing temperature, the associated $e / C_{g}$ conductance oscillations disappear smoothly to give rise to the $4 e / C_{g}$ oscillations. The smoothness of the crossover suggests that the Fabry-Pérot-like oscillations also occur in a regime where electrons are correlated. This smooth change of periodicity bears similarities but also differences compared to the SU(4) Kondo effect in carbon nanotubes, occurring in the weak tunneling regime $E_{C} \gg \hbar \Gamma>k_{B} T$ $[18,38]$. In the Kondo effect, the tunneling coupling is low enough compared to the charging energy to allow full localization of the charge within the dot, but it is large enough compared to the Kondo energy to enable both spin (i) We measure a fourfold enhancement of the oscillation period of $G_{\text {diff }}\left(V_{g}\right)$ upon decreasing temperature, signaling a crossover from coherent single-electron tunneling to Fabry-Pérot interference; both oscillations coexist at the crossover temperature. (ii) Upon increasing the sourcedrain bias at low temperature, both oscillations coexist over a large bias range. (iii) The Sagnac-like modulation pinpoints the quantum interference nature of the FabryPérot oscillations at zero bias. (iv) The magnetic field data suggest a fourfold spin and orbital degeneracy at zeromagnetic field.

The unexpected temperature-induced crossover, possibly related to charge, spin, and valley fluctuations, raises an important question: How does the strength of charge fluctuations compare to that of spin and valley fluctuations in our experiment? Indeed, when the electron transmission approaches one in open fermion channels, the electron shot noise is suppressed to zero [39], indicating that there are no longer any charge, spin, and valley fluctuations in nanotubes; by contrast, in the lower $\Gamma$ limit of SU(4) Kondo, spin and valley fluctuate, but not the charge. It is then natural to ask how the crossover temperature in our devices compares with the well-known Kondo temperature of closed quantum dots. However, a quantitative description 
of our experiment constitutes a theoretical challenge. It will be interesting to measure shot noise [40-43] and the backaction of the electromechanical coupling [44,45] to further characterize these correlated Fabry-Pérot oscillations.

We thank B. Thibeault at UCSB for fabrication help, W. J. Liang, P. Recher, D. Mantelli, and F. Dolcini for discussions. This work is supported by ERC advanced Grant No. 692876, the Cellex Foundation, the CERCA program from the Generalitat de Catalunya, AGAUR (Grant No. 2017SGR1664), Severo Ochoa (Grant No. SEV-2015-0522), MICINN Grant No. RTI2018097953-B-I00 and the Fondo Europeo de Desarrollo Regional. We acknowledge support by the Deutsche Forschungsgemeinschaft within SFB 1277 B04.

[1] S. Datta, Electronic Transport in Mesoscopic Systems (Cambridge University Press, Cambridge, England, 1995).

[2] L. P. Kouwenhoven, C. M. Marcus, P. L. McEuen, S. Tarucha, R. M. Westervelt, and N. S. Wingreen, in Mesoscopic Electron Transport (Springer, Dordrecht, 1997), Chap. Electron transport in quantum dots, pp. 105-214.

[3] S. J. Tans, M. H. Devoret, H. Dai, A. Thess, R. E. Smalley, L. J. Geerligs, and C. Dekker, Individual single-wall carbon nanotubes as quantum wires, Nature (London) 386, 474 (1997).

[4] M. Bockrath, D. H. Cobden, P. L. McEuen, N. G. Chopra, A. Zettl, A. Thess, and R. E. Smalley, Single-electron transport in ropes of carbon nanotubes, Science 275, 1922 (1997).

[5] S. De Franceschi, J. A. van Dam, E. P. A. M. Bakkers, L. F. Feiner, L. Gurevich, and L. P. Kouwenhoven, Singleelectron tunneling in InP nanowires, Appl. Phys. Lett. 83, 344 (2003).

[6] V. V. Deshpande and M. Bockrath, The one-dimensional Wigner crystal in carbon nanotubes, Nat. Phys. 4, 314 (2008).

[7] S. Pecker, F. Kuemmeth, A. Secchi, M. Rontani, D. C. Ralph, P. L. McEuen, and S. Ilani, Observation and spectroscopy of a two-electron Wigner molecule in an ultraclean carbon nanotube, Nat. Phys. 9, 576 (2013).

[8] I. Shapir, A. Hamo, S. Pecker, C. P. Moca, Ö. Legeza, G. Zarand, and S. Ilani, Imaging the electronic Wigner crystal in one dimension, Science 364, 870 (2019).

[9] N. Lotfizadeh, D. R. McCulley, M. J. Senger, H. Fu, E. D. Minot, B. Skinner, and V. V. Deshpande, Band-GapDependent Electronic Compressibility of Carbon Nanotubes in the Wigner Crystal Regime, Phys. Rev. Lett. 123, 197701 (2019).

[10] J. Nygård, D. H. Cobden, and P. E. Lindelof, Kondo physics in carbon nanotubes, Nature (London) 408, 342 (2000).

[11] P. Jarillo-Herrero, J. Kong, H. S. J. van der Zant, C. Dekker, L. P. Kouwenhoven, and S. De Franceschi, Orbital Kondo effect in carbon nanotubes, Nature (London) 434, 484 (2005).
[12] J. Paaske, A. Rosch, P. Wölfle, N. Mason, C. M. Marcus, and J. Nygård, Non-equilibrium singlet-triplet Kondo effect in carbon nanotubes, Nat. Phys. 2, 460 (2006).

[13] A. Makarovski, J. Liu, and G. Finkelstein, Evolution of Transport Regimes in Carbon Nanotube Quantum Dots, Phys. Rev. Lett. 99, 066801 (2007).

[14] T.-F. Fang, W. Zuo, and H.-G. Luo, Kondo Effect in Carbon Nanotube Quantum Dots with Spin-Orbit Coupling, Phys. Rev. Lett. 101, 246805 (2008).

[15] J. P. Cleuziou, N. V. N'Guyen, S. Florens, and W. Wernsdorfer, Interplay of the Kondo Effect and Strong Spin-Orbit Coupling in Multihole Ultraclean Carbon Nanotubes, Phys. Rev. Lett. 111, 136803 (2013).

[16] D. R. Schmid, S. Smirnov, M. Margańska, A. Dirnaichner, P. L. Stiller, M. Grifoni, A. K. Hüttel, and C. Strunk, Broken SU(4) symmetry in a Kondo-correlated carbon nanotube, Phys. Rev. B 91, 155435 (2015).

[17] M. Niklas, S. Smirnov, D. Mantelli, M. Marganska, N.-V. Nguyen, W. Wernsdorfer, J.-P. Cleuziou, and M. Grifoni, Blocking transport resonances via Kondo many-body entanglement in quantum dots, Nat. Commun. 7, 12442 (2016).

[18] M. Ferrier, T. Arakawa, T. Hata, R. Fujiwara, R. Delagrange, R. Weil, R. Deblock, R. Sakano, A. Oguri, and K. Kobayashi, Universality of non-equilibrium fluctuations in strongly correlated quantum liquids, Nat. Phys. 12, 230 (2016).

[19] M. Ferrier, T. Arakawa, T. Hata, R. Fujiwara, R. Delagrange, R. Deblock, Y. Teratani, R. Sakano, A. Oguri, and K. Kobayashi, Quantum Fluctuations Along Symmetry Crossover in a Kondo-Correlated Quantum Dot, Phys. Rev. Lett. 118, 196803 (2017).

[20] M. M. Desjardins, J. J. Viennot, M. C. Dartiailh, L. E. Bruhat, M. R. Delbecq, M. Lee, M.-S. Choi, A. Cottet, and T. Kontos, Observation of the frozen charge of a Kondo resonance, Nature (London) 545, 71 (2017).

[21] T. S. Jespersen, M. Aagesen, C. Srensen, P. E. Lindelof, and J. Nygård, Kondo physics in tunable semiconductor nanowire quantum dots, Phys. Rev. B 74, 233304 (2006).

[22] W. Liang, M. Bockrath, D. Bozovic, J. H. Hafner, M. Tinkham, and H. Park, Fabry-Perot interference in a nanotube electron waveguide, Nature (London) 411, 665 (2001).

[23] L. V. Litvin, H.-P. Tranitz, W. Wegscheider, and C. Strunk, Decoherence and single electron charging in an electronic Mach-Zehnder interferometer, Phys. Rev. B 75, 033315 (2007).

[24] N. Y. Kim, P. Recher, W. D. Oliver, Y. Yamamoto, J. Kong, and H. Dai, Tomonaga-Luttinger Liquid Features in Ballistic Single-Walled Carbon Nanotubes: Conductance and Shot Noise, Phys. Rev. Lett. 99, 036802 (2007).

[25] A. Dirnaichner, M. del Valle, K. J. G. Götz, F. J. Schupp, N. Paradiso, M. Grifoni, C. Strunk, and A. K. Hüttel, Secondary Electron Interference from Trigonal Warping in Clean Carbon Nanotubes, Phys. Rev. Lett. 117, 166804 (2016).

[26] N. Lotfizadeh, M. J. Senger, D. R. McCulley, E. D. Minot, and V. V. Deshpande, Sagnac electron interference as a probe of electronic structure, arXiv:1808.01341.

[27] A. V. Kretinin, R. Popovitz-Biro, D. Mahalu, and H. Shtrikman, Multimode Fabry-Pérot conductance oscillations in suspended stacking-faults-free InAs nanowires, Nano Lett. 10, 3439 (2010). 
[28] I. Neder, M. Heiblum, Y. Levinson, D. Mahalu, and V. Umansky, Unexpected Behavior in a Two-Path Electron Interferometer, Phys. Rev. Lett. 96, 016804 (2006).

[29] E. A. Laird, F. Kuemmeth, G. A. Steele, K. GroveRasmussen, J. Nygård, K. Flensberg, and L.P. Kouwenhoven, Quantum transport in carbon nanotubes, Rev. Mod. Phys. 87, 703 (2015).

[30] J. Cao, Q. Wang, and H. Dai, Electron transport in very clean, as-grown suspended carbon nanotubes, Nat. Mater. 4, 745 (2005).

[31] See Supplemental Material at http://link.aps.org/ supplemental/10.1103/PhysRevLett.125.187701 for additional discussion on current annealing, transport measurements, and the theoretical work on single-particle Fabry-Perot interference and transport with interactions, which includes Refs. [32-37].

[32] A. Noury, J. Vergara-Cruz, P. Morfin, B. Plaçais, M. C. Gordillo, J. Boronat, S. Balibar, and A. Bachtold, Layering Transition in Superfluid Helium Adsorbed on a Carbon Nanotube Mechanical Resonator, Phys. Rev. Lett. 122, 165301 (2019).

[33] T. S. Jespersen, K. Grove-Rasmussen, K. Flensberg, J. Paaske, K. Muraki, T. Fujisawa, and J. Nygård, Gate-Dependent Orbital Magnetic Moments in Carbon Nanotubes, Phys. Rev. Lett. 107, 186802 (2011).

[34] Y. Meir, N. S. Wingreen, and P. A. Lee, Low-Temperature Transport Through a Quantum Dot: The Anderson Model Out of Equilibrium, Phys. Rev. Lett. 70, 2601 (1993).

[35] Y. Meir and N.S. Wingreen, Landauer Formula for the Current Through an Interacting Electron Region, Phys. Rev. Lett. 68, 2512 (1992).

[36] H. Bruus and K. Flensberg, Many-Body Quantum Theory in Condensed Matter Physics (Oxford Graduate Texts, Oxford, 2005).
[37] M. Lavagna, Transport through an interacting quantum dot driven out-of-equilibrium, J. Phys. Conf. Ser. 592, 012141 (2015).

[38] F. B. Anders, D. E. Logan, M. R. Galpin, and G. Finkelstein, Zero-Bias Conductance in Carbon Nanotube Quantum Dots, Phys. Rev. Lett. 100, 086809 (2008).

[39] Ya. M. Blanter and M. Büttiker, Shot noise in mesoscopic conductors, Phys. Rep. 336, 1 (2000).

[40] E. Onac, F. Balestro, B. Trauzettel, C. F. J. Lodewijk, and L. P. Kouwenhoven, Shot-Noise Detection in a Carbon Nanotube Quantum Dot, Phys. Rev. Lett. 96, 026803 (2006).

[41] P. Recher, N. Y. Kim, and Y. Yamamoto, TomonagaLuttinger liquid correlations and Fabry-Perot interference in conductance and finite-frequency shot noise in a singlewalled carbon nanotube, Phys. Rev. B 74, 235438 (2006).

[42] F. Wu, P. Queipo, A. Nasibulin, T. Tsuneta, T. H. Wang, E. Kauppinen, and P. J. Hakonen, Shot Noise with Interaction Effects in Single-Walled Carbon Nanotubes, Phys. Rev. Lett. 99, 156803 (2007).

[43] L. G. Herrmann, T. Delattre, P. Morfin, J.-M. Berroir, B. Plaçais, D. C. Glattli, and T. Kontos, Shot Noise in FabryPerot Interferometers Based on Carbon Nanotubes, Phys. Rev. Lett. 99, 156804 (2007).

[44] C. Urgell, W. Yang, S. L. De Bonis, C. Samanta, M. J. Esplandiu, Q. Dong, Y. Jin, and A. Bachtold, Cooling and self-oscillation in a nanotube electromechanical resonator, Nat. Phys. 16, 32 (2020).

[45] Y. Wen, N. Ares, F. J. Schupp, T. Pei, G. A. D. Briggs, and E. A. Laird, A coherent nanomechanical oscillator driven by single-electron tunnelling, Nat. Phys. 16, 75 (2020). 